\title{
Effectiveness and implementation of models of cancer survivorship care: an overview of systematic reviews
}

\author{
Raymond J. Chan 1,2,3 (1) · Fiona Crawford-Williams ${ }^{1,3} \cdot$ Megan Crichton $^{3,4} \cdot$ Ria Joseph $^{3} \cdot$ Nicolas H. Hart $^{1,3,5,6}$. \\ Kristi Milley ${ }^{7,8} \cdot$ Paige Druce $^{7,8} \cdot$ Jianrong Zhang ${ }^{8} \cdot$ Michael Jefford $^{9,10} \cdot$ Karolina Lisy $^{9,10} \cdot$ Jon Emery ${ }^{7,8}$. \\ Larissa Nekhlyudov ${ }^{11,12}$
}

Received: 8 September 2021 / Accepted: 27 October 2021 / Published online: 16 November 2021

(c) The Author(s), under exclusive licence to Springer Science+Business Media, LLC, part of Springer Nature 2021

\begin{abstract}
Purpose To critically assess the effectiveness and implementation of different models of post-treatment cancer survivorship care compared to specialist-led models of survivorship care assessed in published systematic reviews.

Methods MEDLINE, CINAHL, Embase, and Cochrane CENTRAL databases were searched from January 2005 to May 2021. Systematic reviews that compared at least two models of cancer survivorship care were included. Article selection, data extraction, and critical appraisal were conducted independently by two authors. The models were evaluated according to cancer survivorship care domains, patient and caregiver experience, communication and decision-making, care coordination, quality of life, healthcare utilization, costs, and mortality. Barriers and facilitators to implementation were also synthesized. Results Twelve systematic reviews were included, capturing 53 primary studies. Effectiveness for managing survivors' physical and psychosocial outcomes was found to be no different across models. Nurse-led and primary care provider-led models may produce cost savings to cancer survivors and healthcare systems. Barriers to the implementation of different models of care included limited resources, communication, and care coordination, while facilitators included survivor engagement, planning, and flexible services.

Conclusions Despite evidence regarding the equivalent effectiveness of nurse-led, primary care-led, or shared care models, these models are not widely adopted, and evidence-based recommendations to guide implementation are required. Further research is needed to address effectiveness in understudied domains of care and outcomes and across different population groups.

Implications for Cancer Survivors Rather than aiming for an optimal "one-size fits all" model of survivorship care, applying the most appropriate model in distinct contexts can improve outcomes and healthcare efficiency.
\end{abstract}

Keywords Cancer survivorship $\cdot$ Post-treatment follow-up $\cdot$ Models of care $\cdot$ Systematic review $\cdot$ Shared care $\cdot$ Nurse-led care $\cdot$ Primary care led

\section{Background}

As the number of cancer survivors increases worldwide, there have been many efforts to define and advocate for quality survivorship care, with the ultimate aim to improve survival, physical symptom management, psychosocial effects, and quality of life of cancer survivors [1-3]. There have been calls over the last few decades to improve the quality of survivorship care and promote the integration of

Raymond J. Chan

raymond.chan@flinders.edu.au

Extended author information available on the last page of the article primary care providers [4], and several models of follow-up post-treatment care for cancer survivors have been debated [4-8]. The model of care concept refers to the planning and delivery of health services (i.e., how care is organized and who delivers the care) [9]. Models of cancer survivorship care for post-treatment follow-up can be broadly categorized into specialist-led care, shared care, primary care providerled care, and nurse-led care [9].

The traditional specialist-led model of care continues to be the most prevalent and refers to medically focused (i.e., oncologist or hematologist led) care delivered in the hospital or specialist cancer care setting [10]. While this model ensures patients receive care provided by specialized 
providers, it places a high burden on the acute cancer care system. With a heavy focus on the detection of recurrence or new cancers and limited workforce resources, the sustainability of traditional specialist-led models of care has been repeatedly questioned; such models may also fail to comprehensively meet cancer survivors' supportive care needs [11]. Shared care refers to a collaboration between the specialist/ hospital-based oncology team and a primary care provider, capitalizing on the complementary expertise of various healthcare providers [12]. Primary care provider-led models refer to care provided predominantly in the primary setting. Finally, nurse-led models of care are managed by specialist cancer nurses across multiple settings (hospital, community, etc.) $[13,14]$. In addition, a combination of these models of care may be employed using a variety of delivery methods and may include patient-initiated management or self-management where the cancer survivor coordinates their own follow-up care [5, 15].

Regardless of the care model, comprehensive and quality survivorship care should include surveillance for cancer recurrence; screening for secondary cancers; monitoring for late effects of cancer and its treatment; addressing the risk factors for cancer incidence and mortality such as smoking, weight management, and depression; and coordination between healthcare providers $[2-4,16,17]$. Since the seminal IOM report, national strategies highlighting the important elements of cancer survivorship care, such as psychosocial and supportive care needs, have been developed in several countries $[11,18]$. Although there is some consensus on the core components of post-treatment cancer care, current models of care are largely dependent on provider preference and existing service delivery mechanisms, as well as the design, capacity, and funding available for services $[5,10]$.

It is critical that the evidence regarding the effectiveness and characteristics of various alternative models other than specialist-led models across cancer survivorship outcomes is properly considered in determining the appropriate model of care for the patient in a given setting. Furthermore, it is important for the cancer survivorship community to reflect on how other models of care beyond specialist-led care may better address unmet needs of cancer survivors and achieve quality survivorship care, potentially with more efficient use of services $[19,20]$. This overview of systematic reviews (SRs) provides a critical synthesis of level 1 evidence on the effectiveness and implementation of nurse-led, primary care provider-led, or shared care models of post-treatment followup care in comparison to specialist-led models for cancer survivors across cancer survivorship domains and outcomes.

\section{Methods}

This overview of SRs was conducted in accordance with the Cochrane Handbook for SRs [21] and the PRISMA 2020 Statement [22]. A prospective protocol was registered with the International Prospective Register of SRs (PROSPERO ID: CRD42020213454).

\section{Search strategy}

MEDLINE, CINAHL, Embase, and Cochrane CENTRAL databases were searched for reviews published in English from January 2005 (when the IOM recommendations for quality cancer survivorship care were released) to 21 May 2021. The search strategy used a combination of keywords based on the following terms: cancer AND model of care OR survivorship OR follow-up AND systematic review and is reported in full in Supplementary Material 1. Additional searches using Google Scholar and a manual review of the reference lists of all included articles was conducted to locate SRs not identified in the search strategy.

\section{Eligibility criteria}

Applying the PICO framework, the population of interest included cancer survivors of any age and any stage of cancer who had completed primary cancer treatment. Primary cancer treatment was defined as any modality or multimodality of therapy intended to treat cancer prescribed by a specialist and included continuing maintenance therapies (e.g., surgery, radiotherapy, chemotherapy, hormone therapy, immunotherapy). Cancer survivors receiving end-of-life care were excluded. The intervention of interest was any model of care for post-treatment follow-up in comparison to any other. Our definition of models of care excluded survivorship care interventions which were delivered as a supplement to usual care, such as the inclusion of a survivorship care plan (SCP) or telephone education sessions. Consistent with the definition provided by Taylor et al. [9], models of survivorship care were included if they provided a clear indication of the health professionals responsible for delivering care and stated the care delivery setting. Finally, models of care were only included if they reported on process and effectiveness outcomes relevant to the following aspects of the quality of cancer survivorship care framework defined by Nekhlyudov et al. [2]: (1) domains of care-prevention and surveillance for recurrence and new cancers, surveillance and management of physical effects, surveillance and management of psychosocial effects, surveillance and management of chronic medical conditions, health promotion, and disease prevention; (2) contextual domains - clinical structure, communication/decision-making, care coordination, patient, or 
caregiver experience; and (3) healthcare outcomes-healthrelated quality of life, healthcare utilization, costs of care, and mortality. For this review, domains of care and contextual domains are conceptualized as process outcomes, whereas healthcare outcomes are effectiveness outcomes. Barriers and facilitators to the implementation of models of care were also extracted from included reviews, but these evaluations were not an inclusion criterion. Lastly, SRs were included if they met the following PRISMA-P statement definition of a SR [23]: (A) had an explicit aim; (B) used a systematic search strategy and selection of studies; and (C) systematically synthesized data using narrative synthesis and/or meta-analysis. Unpublished work, abstracts, letters, and conference proceedings were excluded.

\section{Screening and data extraction}

Title and abstract screening followed by full-text screening was shared between all authors and conducted in duplicate. Disagreements were resolved via discussion between authors (P.D., K.M., R.C., M.C., and J.Z.). Extraction of characteristics and findings of included SRs was conducted independently by two authors (M.C. and F.C.) and checked for accuracy by a third author (R.C.). Overall effect estimates and other quantitative data were extracted and presented in tabular format. Where reviews reported data on cancer survivors undergoing treatment or end-of-life care as well as populations in the post-treatment phase, only data relating to cancer survivors in the post-treatment phase were extracted.

\section{Quality appraisal}

Two authors (M.C. and R.J.) independently conducted a systematic review quality assessment using the Assessment of Multiple SRs (AMSTAR) 2 Checklist [24], a 16-question tool that evaluates each item as "yes" or "no" and yields a final overall rating of "high," "moderate," "low," or "critically low" quality. Study quality disagreements were managed by consensus between authors and resolved by discussion with a third author if necessary (RC).

\section{Data synthesis}

Data were synthesized and reported narratively, and no additional re-analysis of outcome data was conducted. Data were presented according to the cancer survivorship domains stated in the research question, with barriers and facilitators related to implementation synthesized narratively.

Primary studies included in more than one SR have been clearly indicated in Supplementary Material 2. The extent to which primary studies overlap in the included SRs has been reported as the corrected covered area (CCA) [25]. CCA has been calculated using the following formula: $C C A=\frac{\mathrm{N}-\mathrm{r}}{\mathrm{rc}-\mathrm{r}}$ whereby $N$ represents the total number of primary studies including double counting of overlapping studies, $r$ is the number of primary studies not including double counting of overlapping studies, and $c$ is the total number of SRs included in this review. The final CCA value is represented as a percentage of overlap [25].

\section{Results}

\section{Characteristics of systematic reviews}

Of 1312 unique records identified; a total of 12 SRs were included in this overview of SRs (Fig. 1). The main reason for exclusion during the full-text review stage was SRs assessing elements of care such as diagnostic test accuracy or frequency of follow-up, rather than the model of care itself (Supplementary Material 3). The 12 SRs captured 53 primary studies that provided data comparing two or more different models of care in post-treatment cancer survivors. The number of primary studies included from each SR ranged from 4 to 17 . A total of 19 of the primary studies were included in two or more SRs (Supplementary Material 2). The CCA value determined a $7.4 \%$ overlap of primary studies in the SRs [25].

Characteristics of the SRs are summarized in Table 1. Eight SRs (67\%) included only randomized controlled trials (RCTs) [26-33], one SR (8\%) included a combination of RCTs and controlled trials [34], and three SRs (25\%) included a combination of RCTs and observational studies [35-37]. Most SRs $(n=10,83 \%)$ [26, 28-30, 32-37] represented mixed cancer types, although one SR focused solely on breast cancer [27], and one focused solely on lung cancer [31]. The most prevalent cancer types in primary studies were breast ( $n=20$ studies, $38 \%)$, gynecological ( $n=6$ studies, $11 \%$ ), colorectal ( $n=5$ studies, $9 \%$ ), prostate ( $n=4$ studies, 7\%), lung ( $n=3$ studies, $6 \%$ ), and esophageal ( $n=3$ studies, $6 \%$ ), with only one study focused on patients with melanoma, chronic lymphocytic leukemia, pancreatic or head and neck cancer, and eight studies $(15 \%)$ in mixed cancer types. Ten SRs (83\%) reported on the number of participants in primary studies, with a total of 24,947 unique patients across all studies. Gender and age were poorly reported in the SRs, although participants from all primary studies were adult cancer survivors. Almost all primary studies were conducted in Western developed countries. The majority were conducted in the UK (16 studies, 30\%), followed by Canada ( $n=8$ studies, $15 \%$ ), the USA ( $n=8$ studies, $15 \%)$, Netherlands ( $n=5$ studies, 9\%), Sweden $(n=5$ studies, 9\%), Australia ( $n=4$ studies, $7 \%)$, Denmark $(n=2$ studies, $4 \%$ ), and Norway ( $n=2$ studies, $4 \%$ ), with one study each from Spain, Japan, and Turkey. 
Fig. 1 PRISMA flowchart for search strategy exploring different models of cancer follow-up care
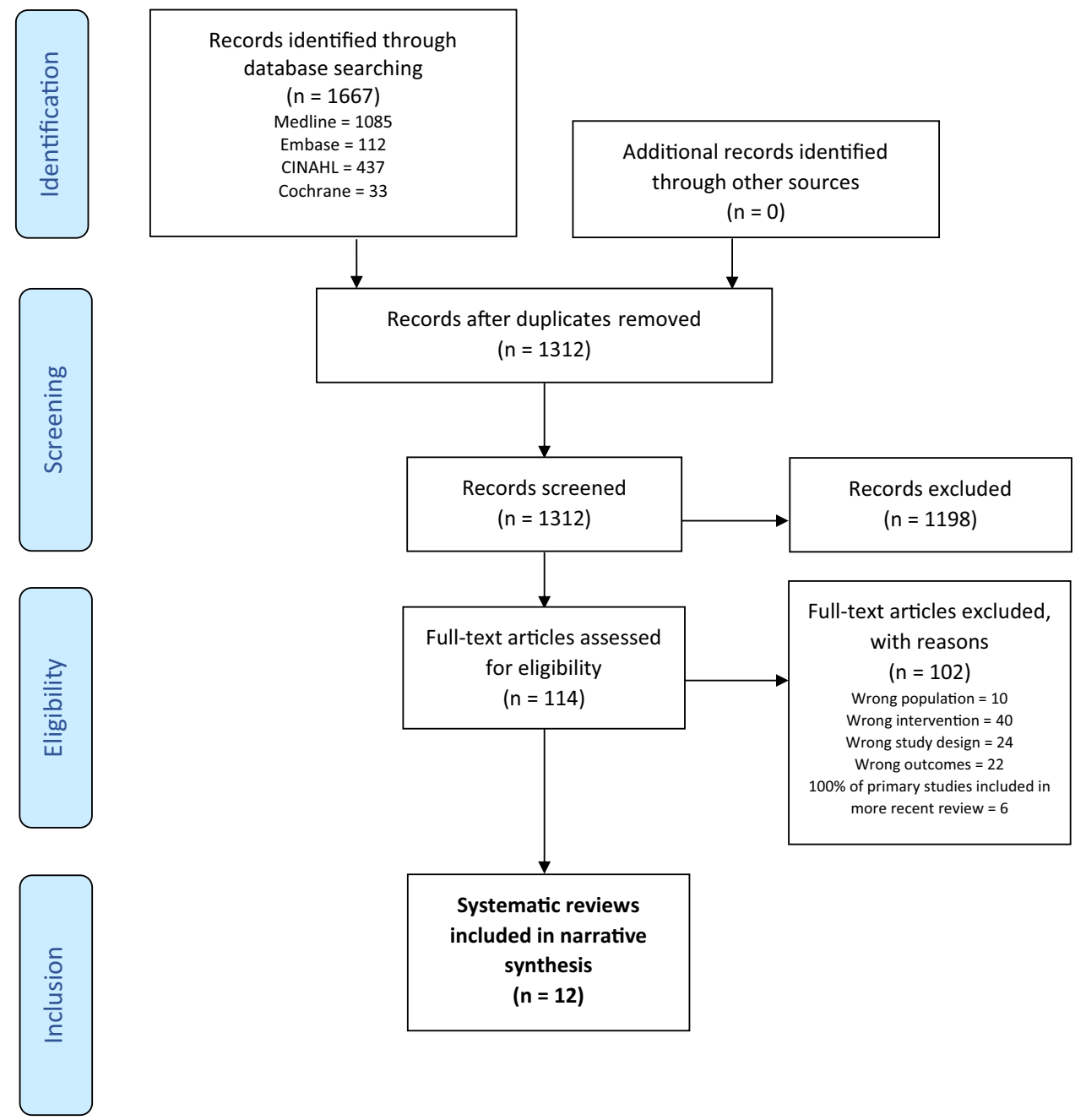

Characteristics of the models of care examined in the SRs are summarized in Table 2. Eight SRs (67\%) evaluated nurse-led models of care [26, 27, 29, 31, 32, 34-36], six SRs $(50 \%)$ evaluated primary care provider models of care [28-30, 35-37], and five SRs (42\%) evaluated shared care (between primary care provider and specialist, or primary care provider and nurse) [28, 30, 32, 33, 37]. Six SRs (50\%) examined multiple models of care within one review. Two SRs (17\%) evaluated patient-initiated models of care focusing on self-management follow-up, whereby cancer survivors were responsible for coordinating their own care on a needs basis and did not receive routine clinical appointments [28, 29]. Conventional medical follow-up care, led by cancer specialists, surgeons, oncologists or hematologists in hospitals, cancer centers, or clinics was the standard care and comparator group in all SRs. These are referred to collectively as specialist-led models throughout the summary of results.

\section{Quality appraisal of systematic reviews}

Quality appraisal results of included SRs using the AMSTAR-2 Tool are presented in Supplementary Material 5. One SR (8\%) was classified as high quality [28], four SRs $(31 \%)$ were rated as moderate quality $[26,29,30,36]$, five SRs (42\%) were rated low quality [27, 32-34, 37], and two SRs $(17 \%)$ were rated critically low quality $[31,35]$. Only one SR provided an explanation for primary study design inclusion (item 3) [26], one provided a list of excluded studies along with explanations for exclusion (item 7) [28], and only one considered sources of funding of primary studies (item 10) [28]. Two SRs [28, 32] specified whether review methods were established prior to conducting the review (item 2).

Nine SRs (75\%) assessed the quality of primary studies using a range of different tools [26-30, 33, 34, 36, 37]. RCTs were assessed using the Cochrane Risk of Bias Tool [26, 28, 32], Critical Appraisal Skills Programme (CASP) 


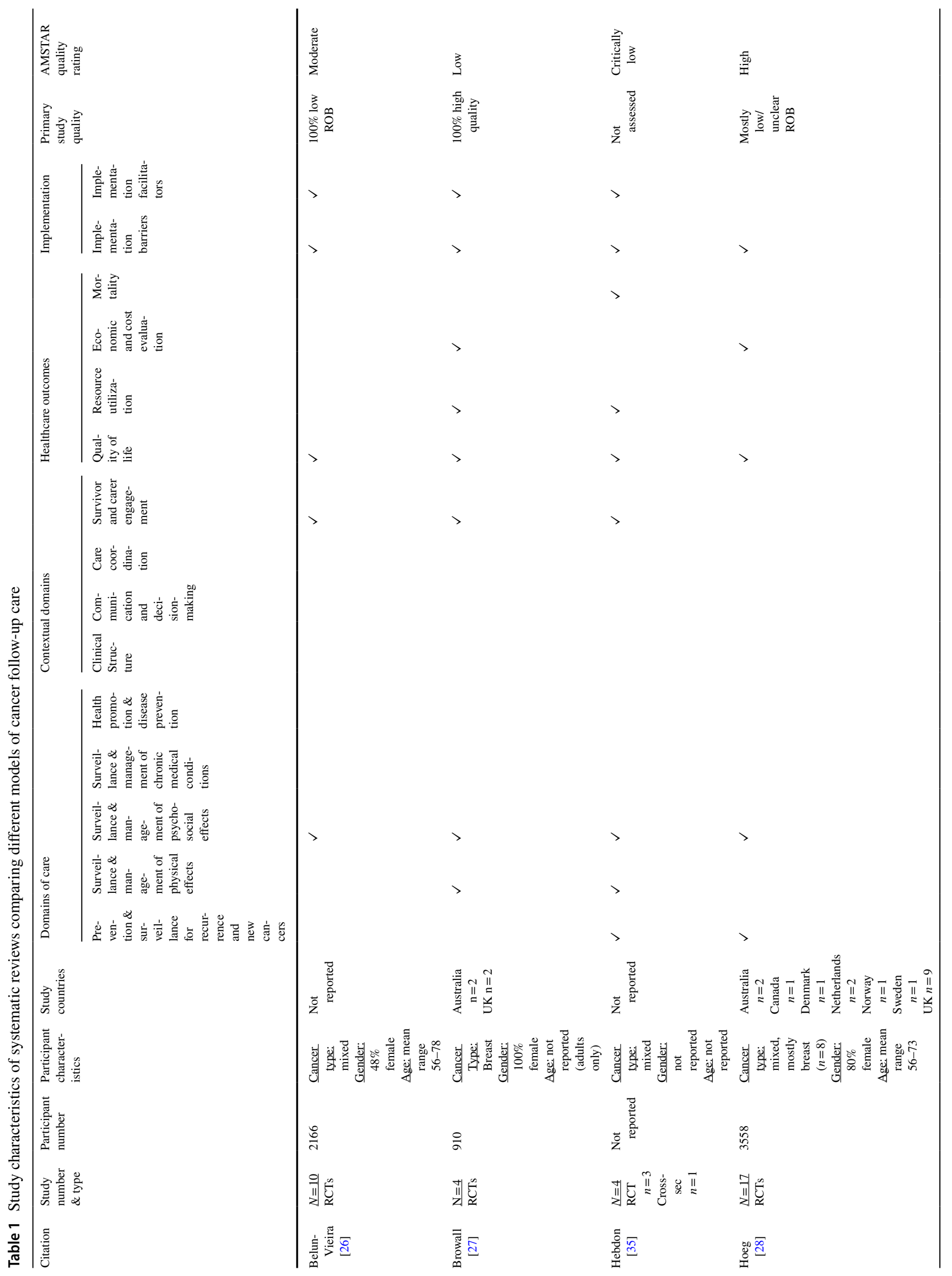




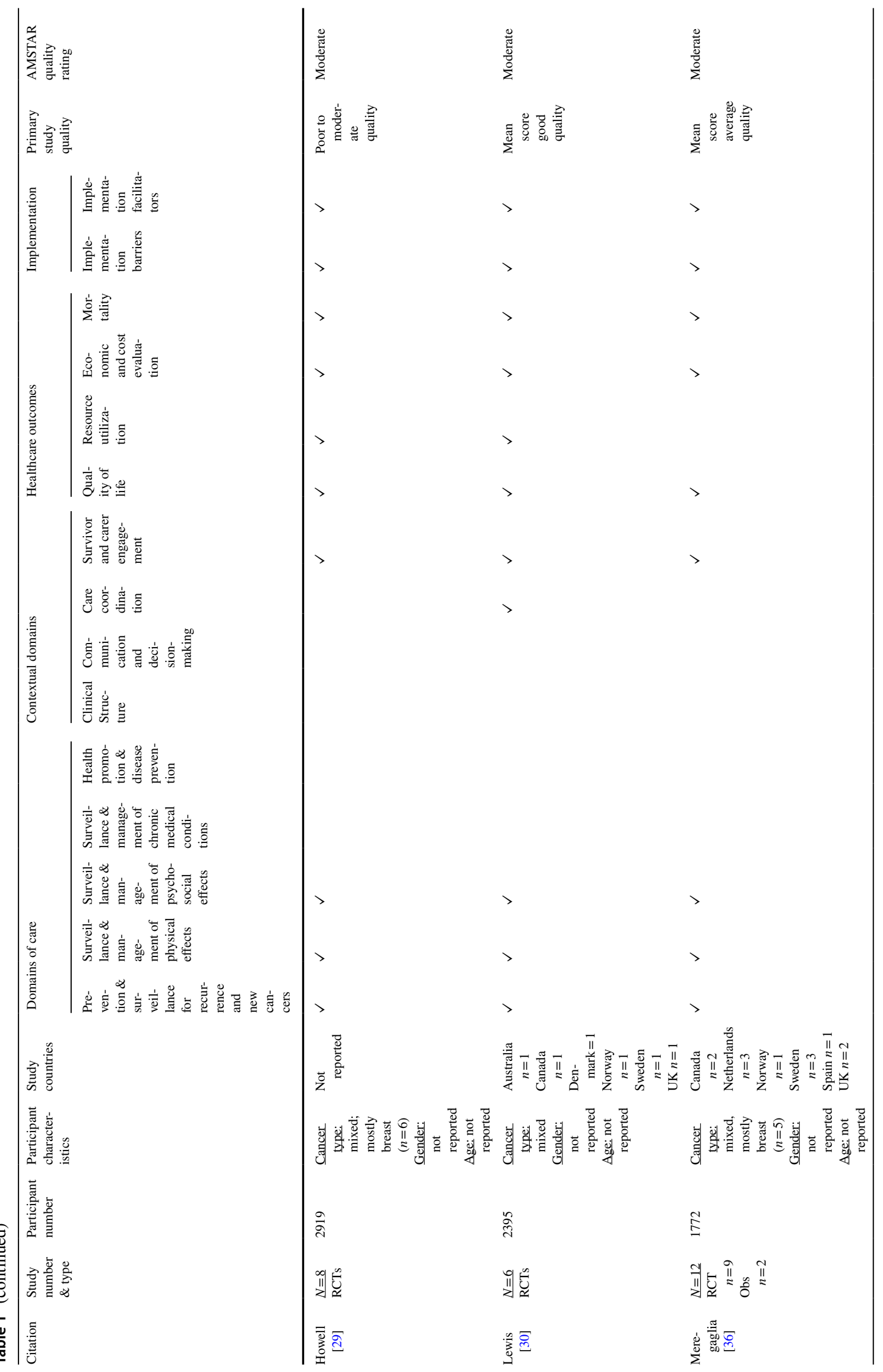




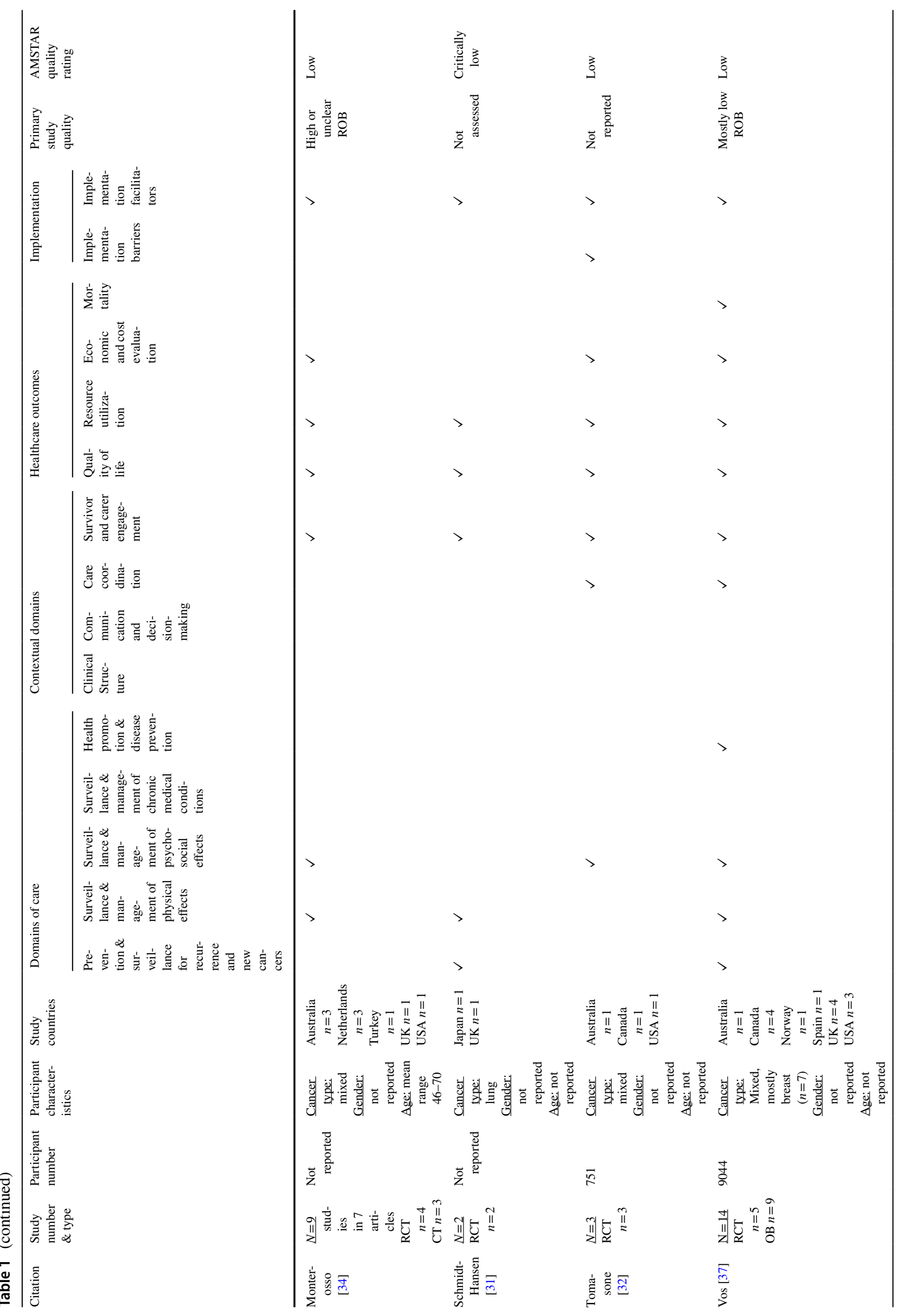




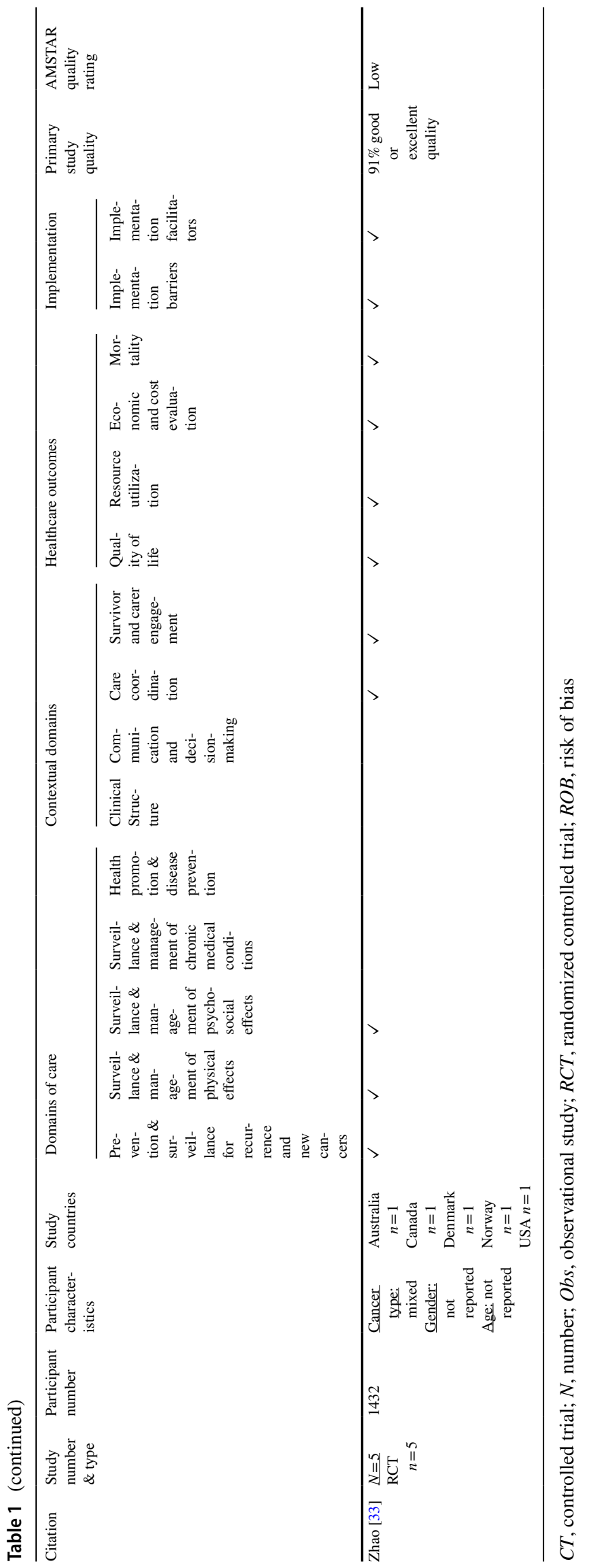


Journal of Cancer Survivorship (2023) 17:197-221

205

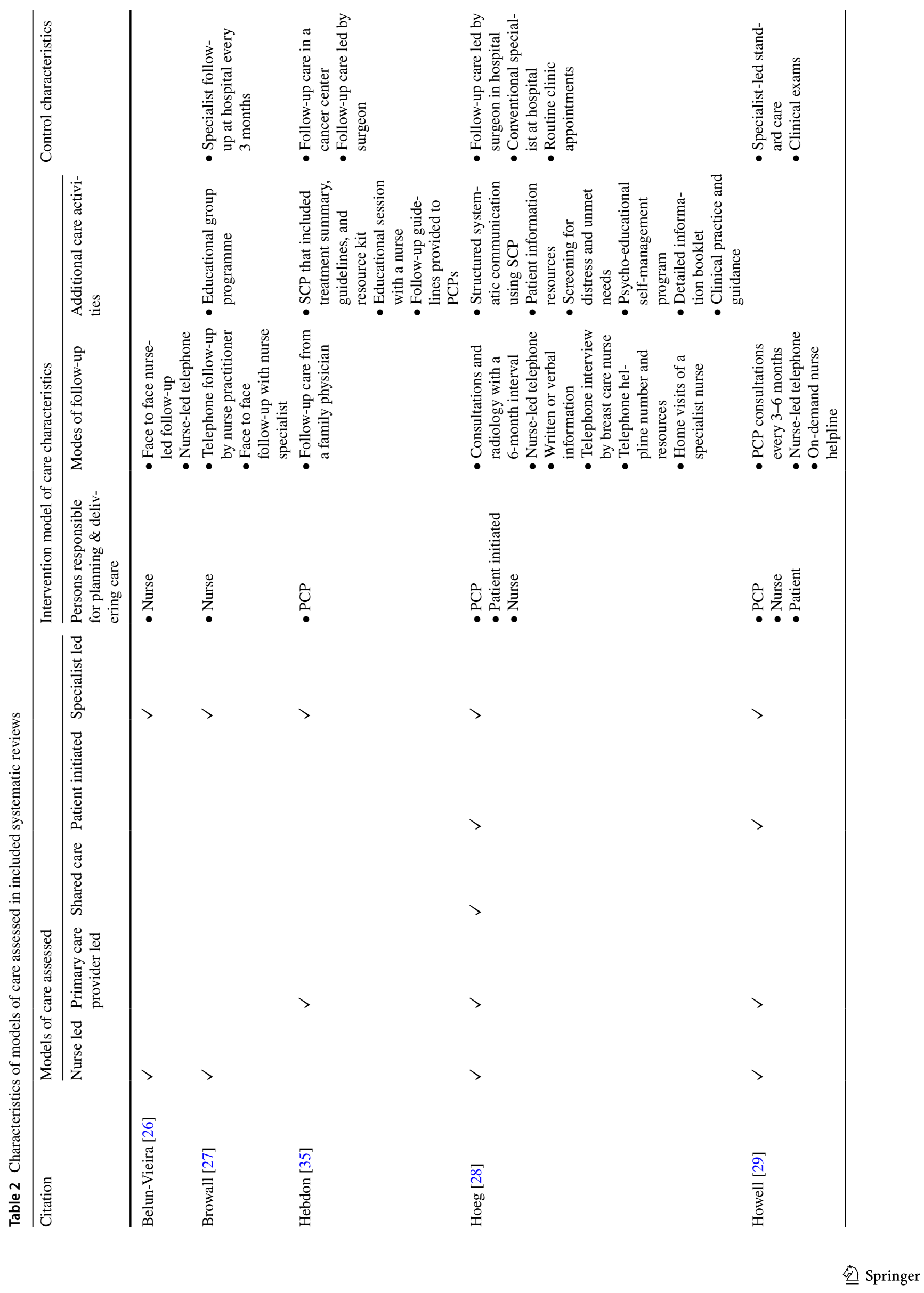




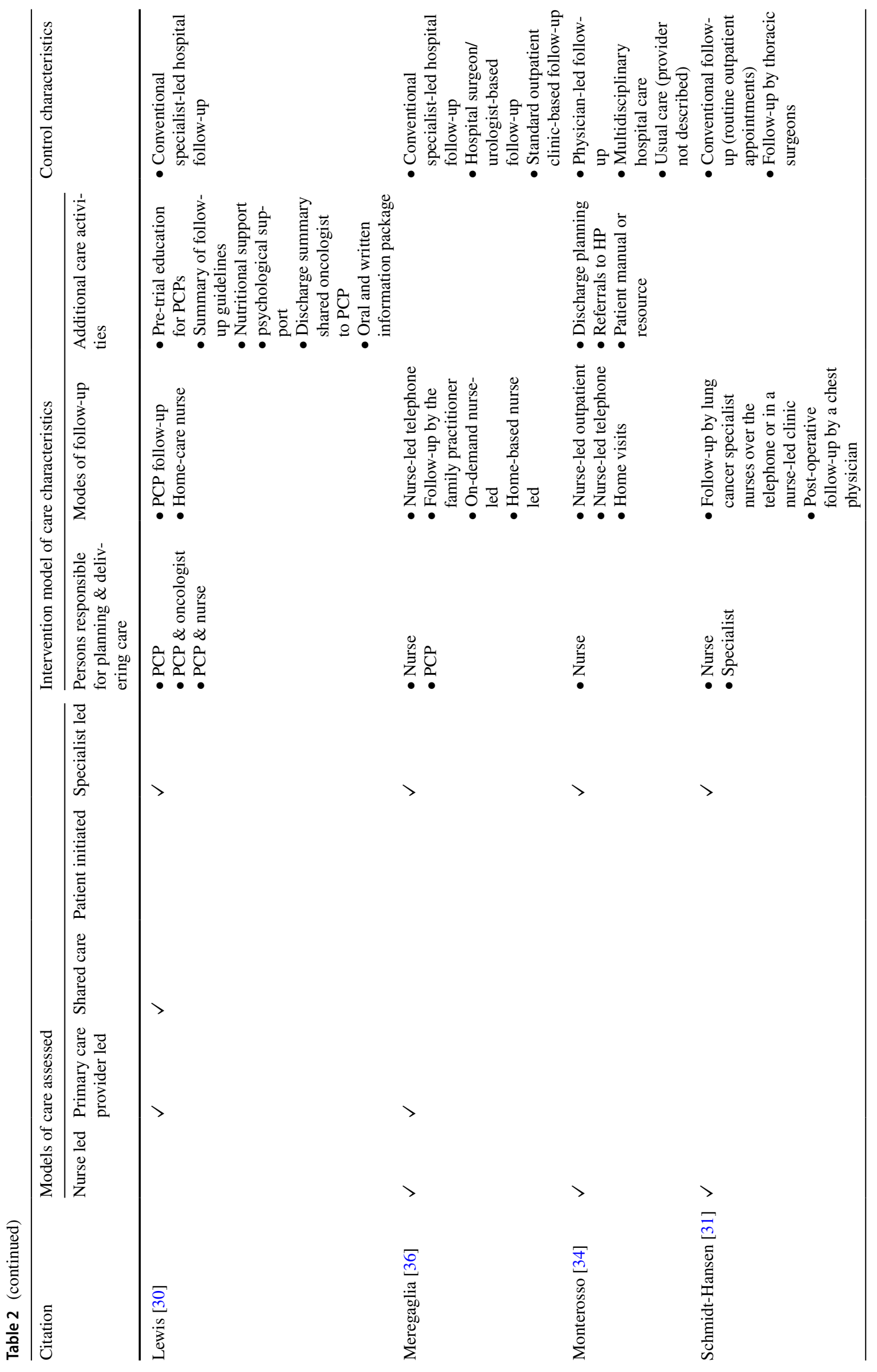




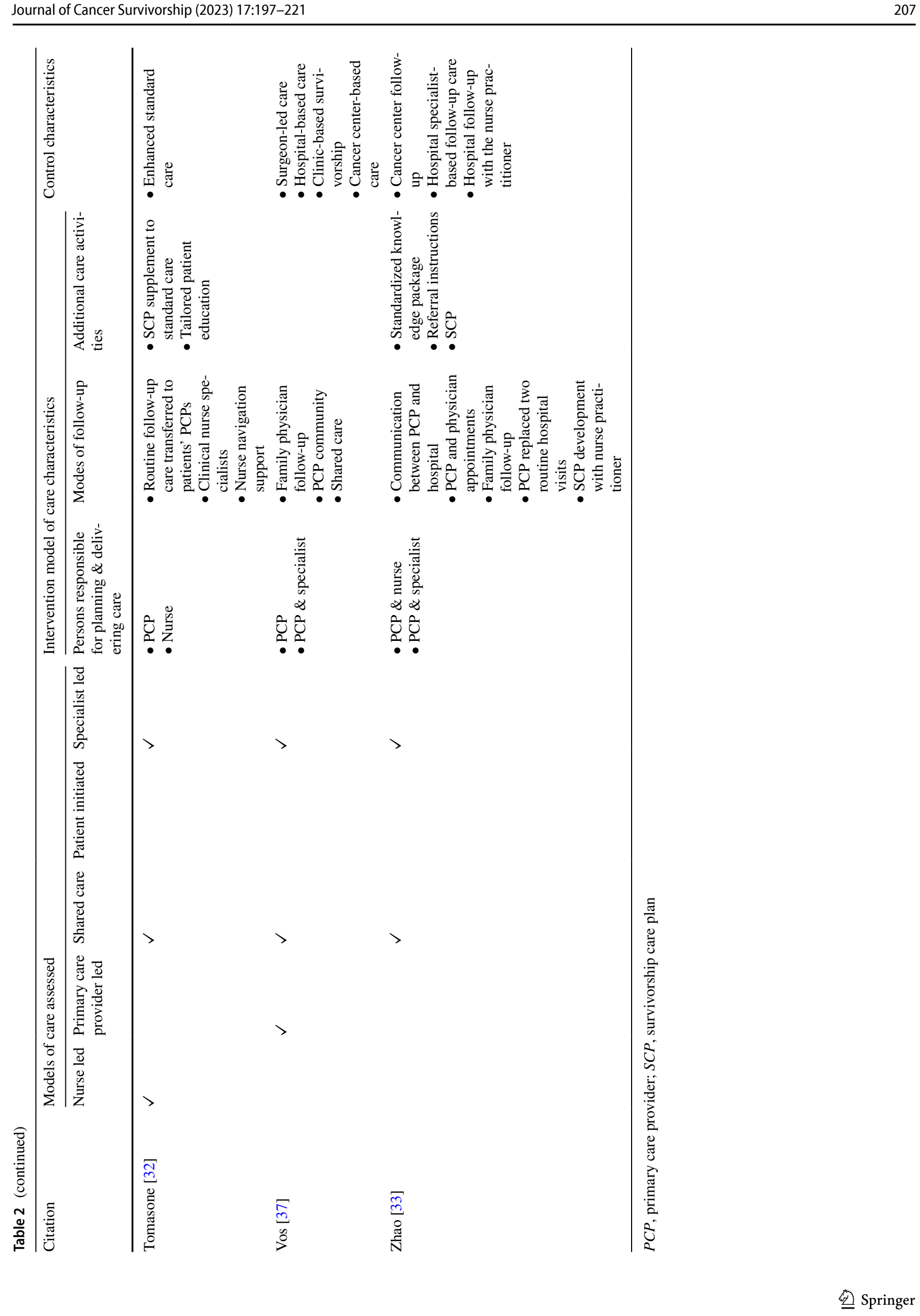


Checklist [27], Scottish Intercollegiate Guidelines Network (SIGN) Checklist [29], Downes and Black Checklist [30], or the Joanna Briggs Institute Checklist [34]. To assess nonrandomized controlled trials, reviews used the Risk of Bias in Non-Randomized Studies of Interventions (ROBINS-I) Tool [37] or the University of Salford Health Care Practice Research and Development Unit (HCPRDU) Evaluation Tool [33]. The Strengthening the Reporting of Observational studies in Epidemiology (STROBE) guidelines were used to evaluate observational studies and the Consolidated Health Economic Evaluation Reporting Standards (CHEERS) checklist was used to assess economic evaluations [36]. Six of the nine SRs $(67 \%)$ reported primary studies as high quality [27, 30, 33] or low risk of bias [26, 35, 37]. The remaining three SRs $(33 \%)$ reported primary studies to be poor to moderate [29] or average quality [36] or having a high or unclear risk of bias [34]. AMSTAR quality ratings and primary study quality are summarized in Table 1 .

\section{Effects of different models of follow-up care}

The findings concerning the effects of nurse-led, primary care provider-led, and shared care models of cancer survivorship care compared with specialist-led care across process and effectiveness outcomes are summarized below. Where two or more SRs reported on the same outcomes, findings are presented in Tables 3 and 4 with the effects presented according to the various models (nurse-led vs primary care provider-led vs shared care). Figure 2 provides a summary of the number of primary studies reporting outcomes relevant to each of the aspects of the quality of the cancer survivorship care framework described by Nekhlyudov et al. [2] More detailed descriptions of the findings reported in each individual SR are presented in Supplementary Material 4.

\section{Effects of models of follow-up care on quality cancer survivorship care domains}

\section{Prevention and surveillance for recurrences and new cancers}

The effects of different models of cancer follow-up care on the prevention and surveillance of recurrences and new cancers was explored in eight SRs (67\%) [28-31, 33, 35-37]. These SRs did not detect statistically significant differences between nurse-led or primary care provider-led care and specialist-led models of care on the incidence of recurrence or the time to detect recurrence. No reviews reported on the impact of models of care on the detection of new primary cancers.

\section{Surveillance and management of physical effects}

The effects of different models of cancer follow-up care on the physical effects of cancer and treatment were explored in nine SRs (75\%), although these were not always the primary endpoints of the primary studies and the range of possible outcomes was limited [27, 29-31, 33-37]. Overall, other models of care did not result in improved patient outcomes when compared with specialist-led models; however, in a limited number of primary studies, fatigue was reported to be reduced in nurse-led and primary care provider-led models compared to specialist led [34, 37]. Additionally, in one SR, appetite loss was reported to be significantly reduced with specialist-led models of care (two primary studies), and pain showed mixed effects (two primary studies) [34].

\section{Surveillance and management of psychosocial effects}

Almost all SRs ( $n=11,92 \%)$ [26-30, 32-37] explored the effects of different models of cancer follow-up care on the psychosocial effects of cancer and its treatment. No statistically significant differences between any models of care compared with specialist-led care were found for anxiety, depression, or psychological distress outcomes [26-30, 32-37]. Cancer survivors' outcomes including fear of cancer recurrence, body image, and unmet supportive care needs were not reported by more than one SR, with inconclusive findings reported.

\section{Surveillance and management of chronic medical conditions}

No SRs explored the effectiveness of different models of care on the surveillance and management of chronic medical conditions, other than cancer itself, such as cardiovascular disease or diabetes.

\section{Health promotion and disease prevention}

Only two primary studies in one SR reported on the effectiveness of different models of cancer follow-up care on health promotion outcomes [37]. The provision of help with lifestyle improvements and discussion of diet among colorectal cancer survivors was found to more likely occur in shared care models in comparison to specialist-led care [38].

\section{Effects of models of cancer follow-up care on healthcare delivery system contextual domains}

\section{Clinical structure}

To assess the effectiveness of clinical structure, studies may have compared the type of healthcare setting (e.g., 
Journal of Cancer Survivorship (2023) 17:197-221

209

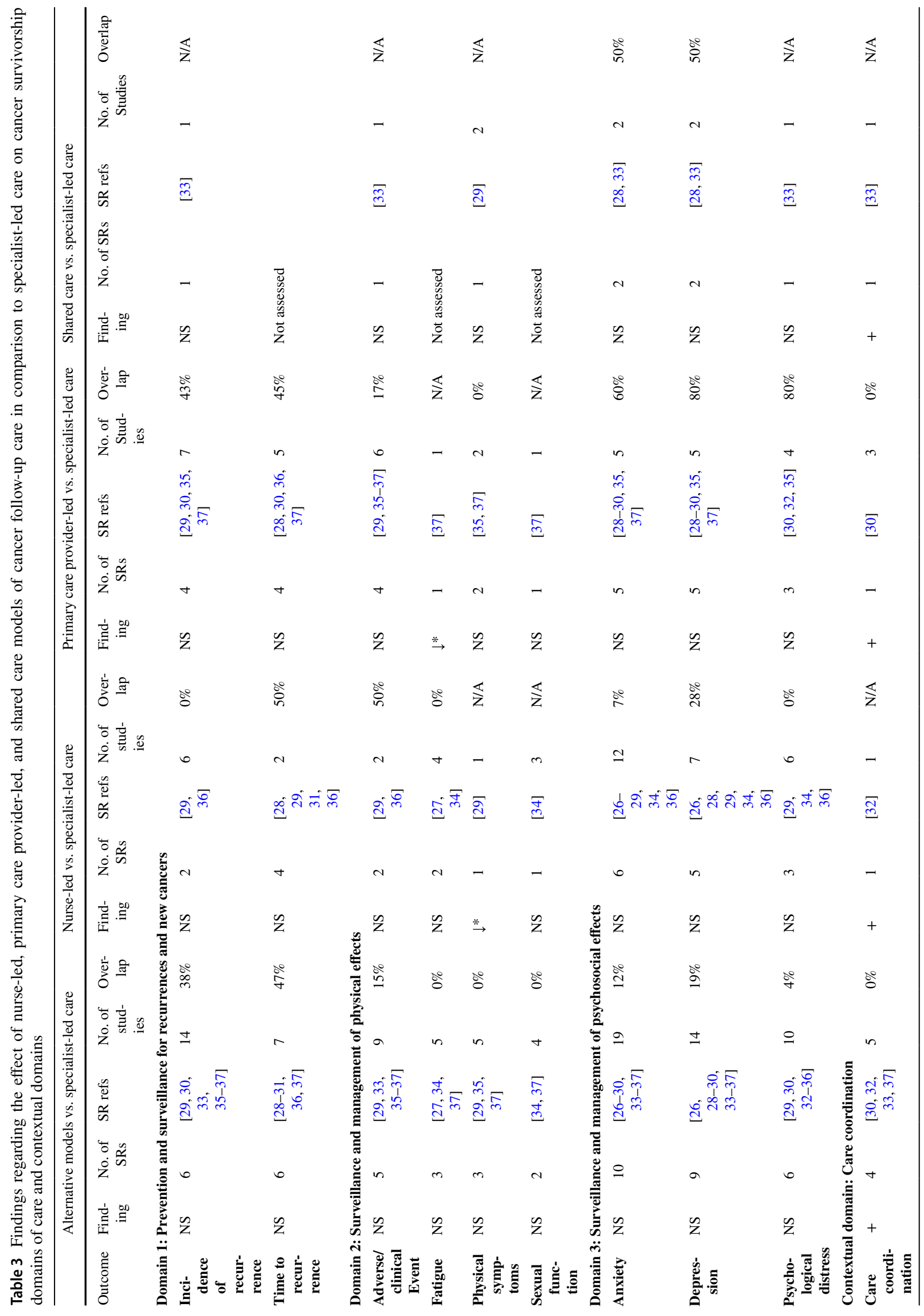

Springer 
survivorship clinic, community-based hospital, academic center, rural, or urban) or whether access to care was adequately enabled (e.g., availability of appointments, navigators, health information technology) within different models of care. Adherence to medical guidelines and confidence in delivering cancer survivorship care was also reported to be higher in primary care provider-led models of follow-up compared with specialist-led care ( $n=1 \mathrm{SR} ; n=1$ primary study) [37]. No SRs explored other effects of the clinical structure on the effectiveness of different models of care, and furthermore, no SRs explored whether opportunities for research trial participation differed according to the different models of care.

\section{Communication and decision-making}

No SRs explored the effectiveness of different models of care on communication or decision-making indicators such as assessment of self-management skills, patient involvement in shared decision-making, or patient-centered communication.

\section{Care coordination}

Four SRs (33\%) reported on outcomes related to the adequacy of care coordination and health providers' engagement [30, 32, 33, 37]. Findings suggest that nurse-led, primary care provider-led, and shared care models can increase primary care involvement in cancer care and improve coordination between primary and secondary care services [30]. The reported care coordination outcomes focused on coordination between providers as we found no SRs that reported on care coordination between patients and providers.

\section{Cancer survivor and caregiver engagement}

Eleven SRs (92\%) reported on outcomes related to the patient experience with care [26, 27, 29-37], all of which reported equivalent or higher patient satisfaction with nurse-led, primary care provider-led, or shared care models compared to specialist-led models of care. Significantly higher patient satisfaction was found with nurse-led models of care relative to specialist-led care $(n=6$ SRs $[26,27,29,31,34,36] ; n=9$ primary studies). Only one primary study considered caregiver satisfaction of care, which was also significantly higher with nurse-led care compared to specialist-led models of care ( $n=1$ SR [34]). 


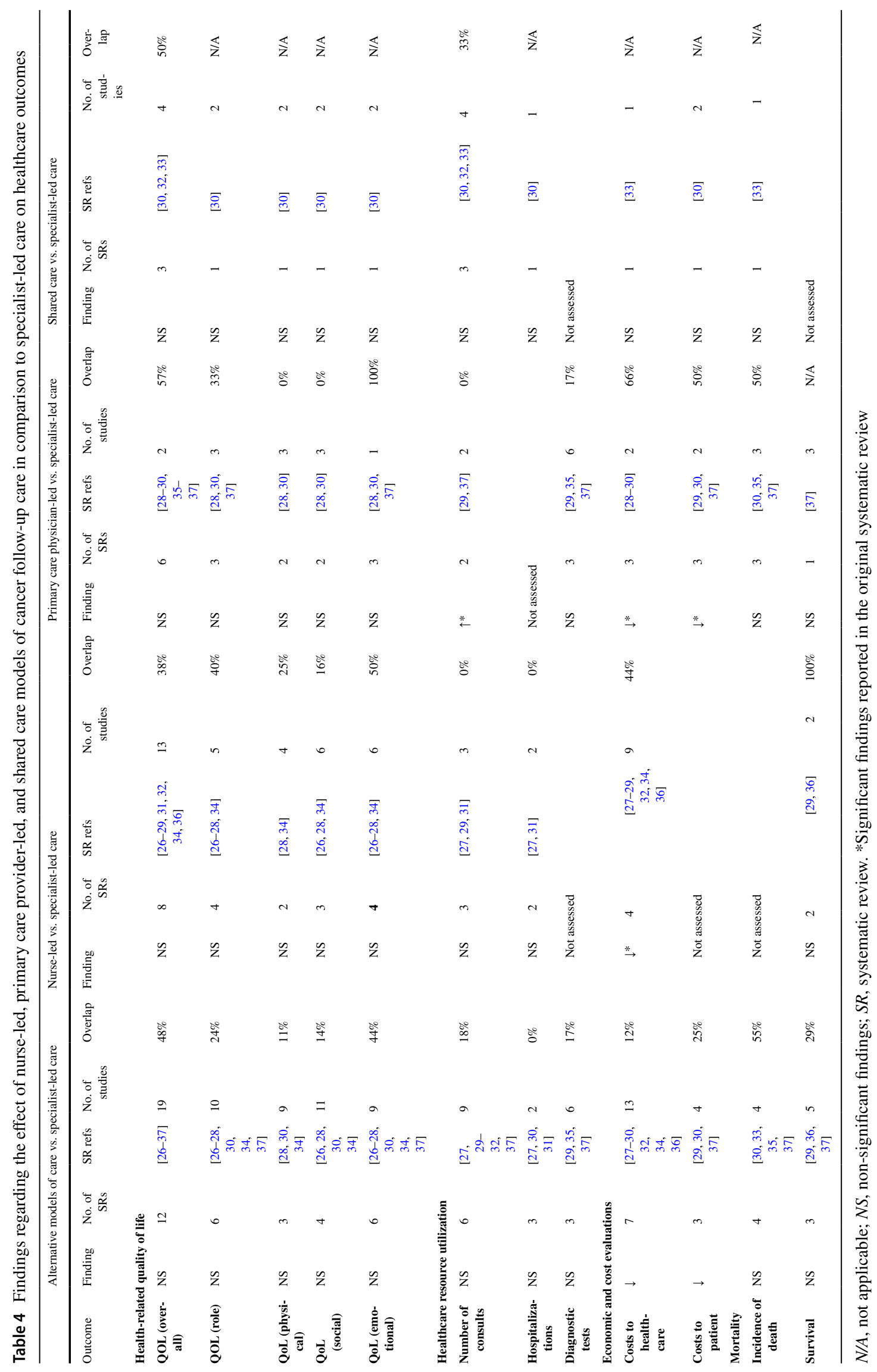




\section{Framework for Measuring Quality of Cancer Survivorship Care}

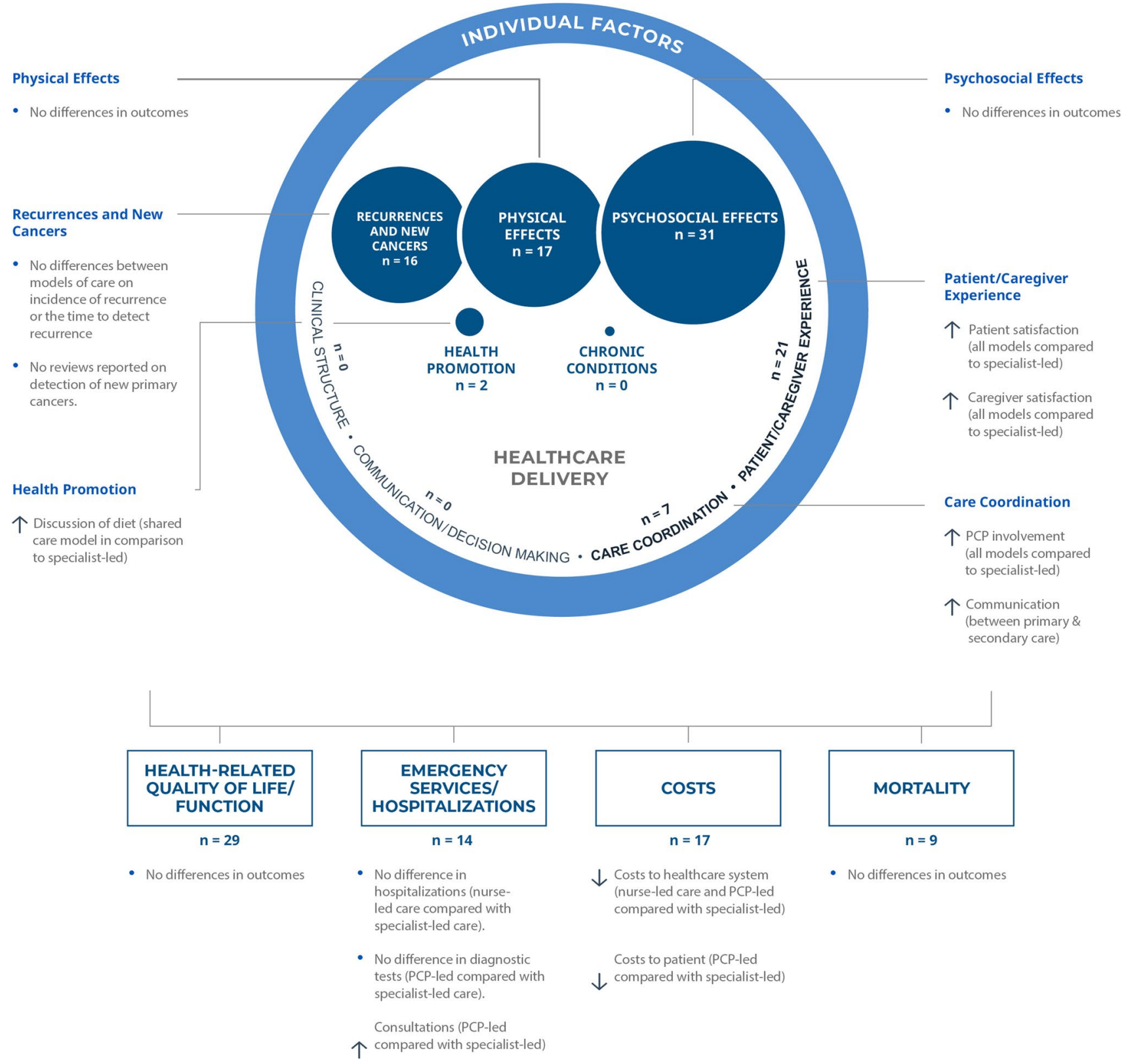

Fig. 2 Primary study numbers and findings according to the cancer survivorship care quality framework

\section{Effects of models of cancer follow-up care on healthcare outcomes}

\section{Health-related quality of life}

All SRs [26-37] explored the effects of different models of cancer follow-up care on health-related quality of life. No significant differences were found between any models of care compared with specialist-led care for overall quality of life as well as the quality of life subscales including role, physical, social, and emotional [26-30, 32-37]; however, one meta-analysis of six studies reported significantly improved overall quality of life for patients in nurse-led care compared with specialist-led care at the 12-month follow-up timepoint [26] (Supplementary Material 4). 


\section{Healthcare resource utilization}

Nine SRs (75\%) explored resource use associated with models of cancer follow-up care [27, 29-35, 37]. The number of consultations was significantly higher with primary care provider-led care compared with specialist-led care; however, there were no differences in the number of consultations between nurse-led care or shared care in comparison with specialist-led models. No statistically significant differences in a number of hospitalizations were detected between nurse-led models of care and specialist-led care, yet this outcome was not assessed in primary care provider-led or shared care models. Finally, there were no overall differences in a number of diagnostic tests detected between primary care provider-led models and specialist-led models reported in the SRs; however, one study did report an increase in diagnostic tests for colorectal patients in primary care-led models [39].

\section{Economic and cost evaluations}

Nine SRs (75\%) explored the costs associated with models of cancer follow-up care [27-30, 32-34, 36, 37]. Overall, costs to the healthcare system were found to be significantly lower with nurse-led and primary care provider-led models of care compared to specialist-led care, but there was no significant difference in costs for the healthcare system for shared care models. Costs to individual cancer survivors were reported to be lower with primary care provider-led models of care compared to specialist-led care, but findings were not significant for reducing patient costs with shared care models. Costs to cancer survivors were not assessed comparing nurse-led models with specialist-led models.

\section{Mortality}

Six SRs compared the effects of different models of followup care on mortality outcomes [29, 30, 33, 35-37]. These SRs did not detect statistically significant differences for incidence of death or survival rates between primary care provider-led care, nurse-led, or shared care models compared with specialist-led care models of follow-up $(n=9$ primary studies).

\section{Implementation of models of cancer follow-up care}

Findings regarding the implementation of different models of cancer follow-up care from SRs are presented in Table 5, with further details provided in Supplementary Material 4. Eleven SRs (92\%) assessed the implementation of different models of care [26, 27, 29-37] and commented on barriers or facilitators which impacted on implementation.

\section{Implementation barriers}

Nine SRs (75\%) reported barriers to implementing various models of care [26-30, 32, 33, 35, 36], summarized in Table 5. Four SRs (33\%) reported that a major barrier to implementing a new model of care was the lack of evidence-based research, which included the lack of quality evidence to suggest which model of care is most effective. The research evidence available to guide implementation of new follow-up care models was described as having small sample sizes, poor data reporting, and a lack of standardized outcomes, methods, and evaluation tools [26, 27, 30, 36]. The issue with generalizing models of care to all cancer populations was highlighted in six SRs (50\%), with patient care needs dependent on cancer type, cancer stage, patient age, and comorbidities, meaning non-specialist care may not be applicable to survivors with more complex needs [26, 27, $29,30,33,36]$. One SR also reported a barrier to the generalizability of models of care relating to the existing evidence coming from developed countries and cities, as findings may not be relevant for developing countries and rural settings [33]. Poor communication between care providers and settings as well as the lack of an informative survivorship care planning tool were also identified as barriers to implementation of models of care in five SRs $(42 \%)$ [28, 30, 32, 33, 35]. Additional barriers reported in two SRs (17\%) related to resources, such as the lack of time to complete survivorship care plans, limited knowledge of cancer survivor issues, and poor adherence to available survivorship guidelines for surveillance, prevention, intervention, and continuity of care $[32,35]$.

\section{Facilitators}

Eleven SRs (92\%) reported facilitators to implementing different models of care [26, 27, 29-37], summarized in Table 5. Facilitators reported in four SRs (33\%) included the utilization of an underpinning theory and thorough assessment of patient needs [26, 27, 29, 31]. Seven SRs (58\%) reported facilitators relating to the characteristics of different models of care such as an increased capability with nonspecialist-led care models to adhere to IOM recommendations or other relevant guidelines, offering alternate delivery methods such as telephone or online, and having a flexible and adaptable service to accommodate individual need [26, 27, 29, 31-33, 35]. Implementation facilitators for communication and care coordination were reported, such as appropriate, effective, and regular communication between care providers, and enhancing communication through the use of a survivorship care planning tool [29, 30, 32, 33, 35], and involvement of multidisciplinary teams and continual professional education in cancer survivorship care [26, 29, 32-35]. 


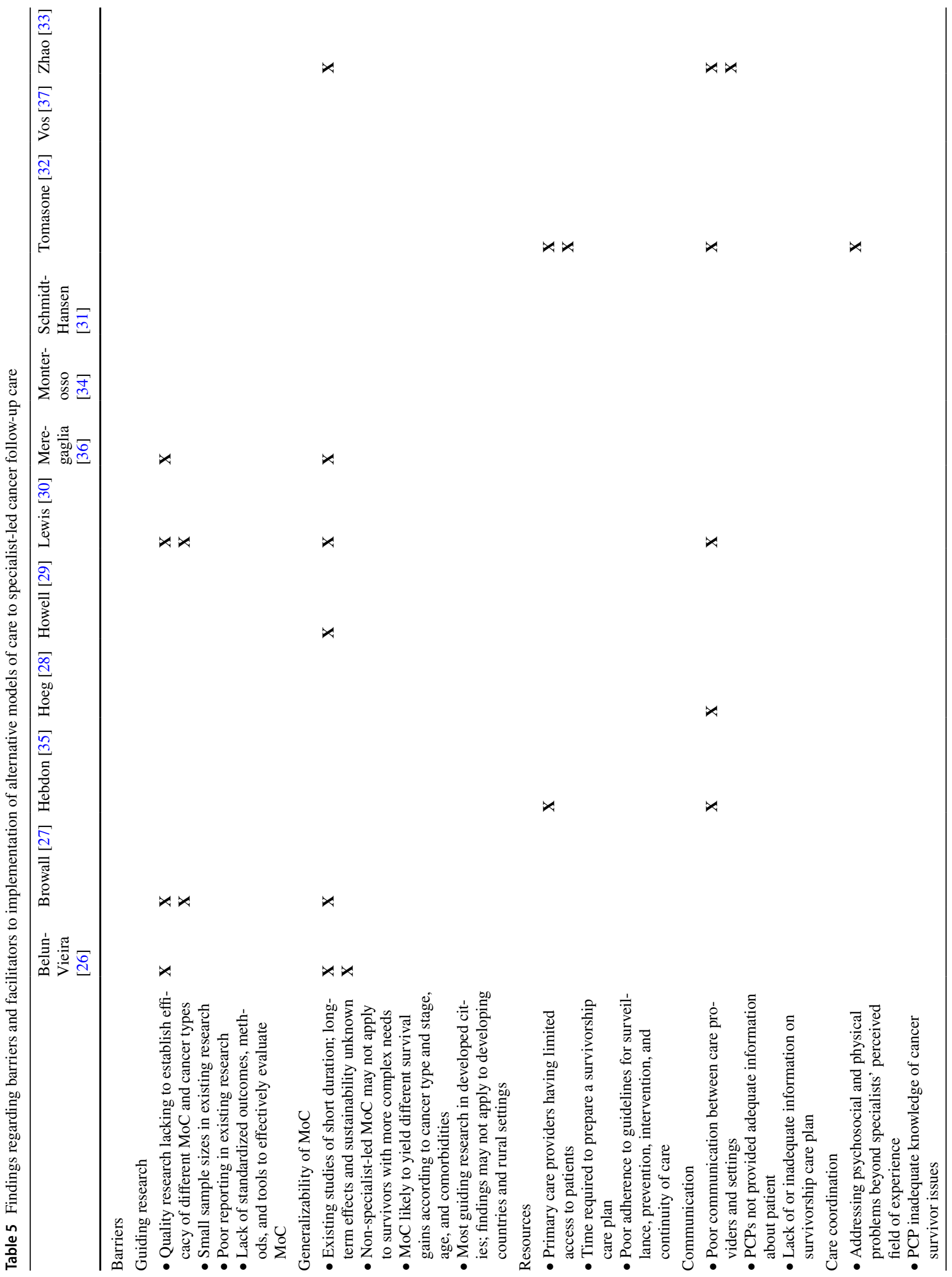




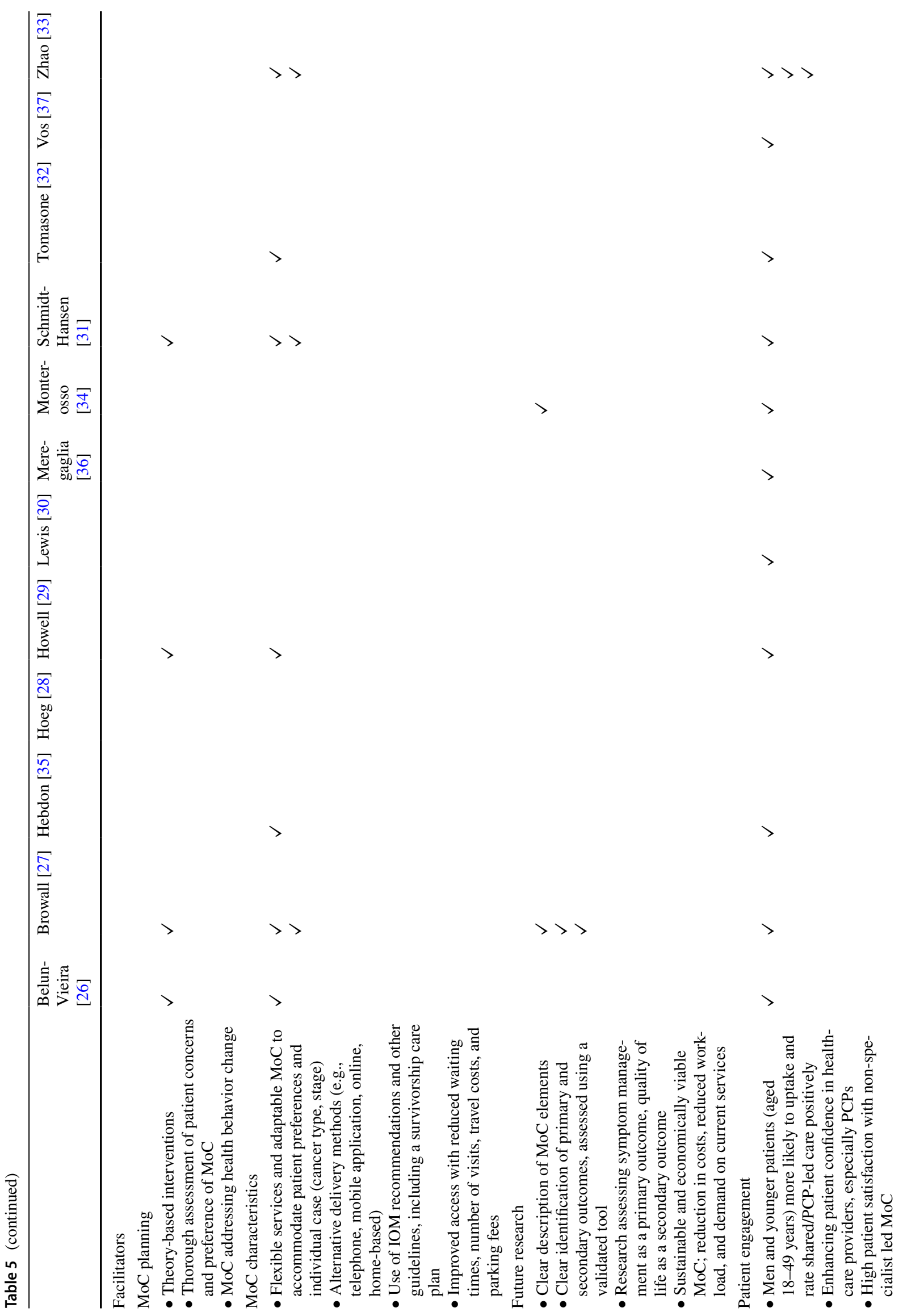




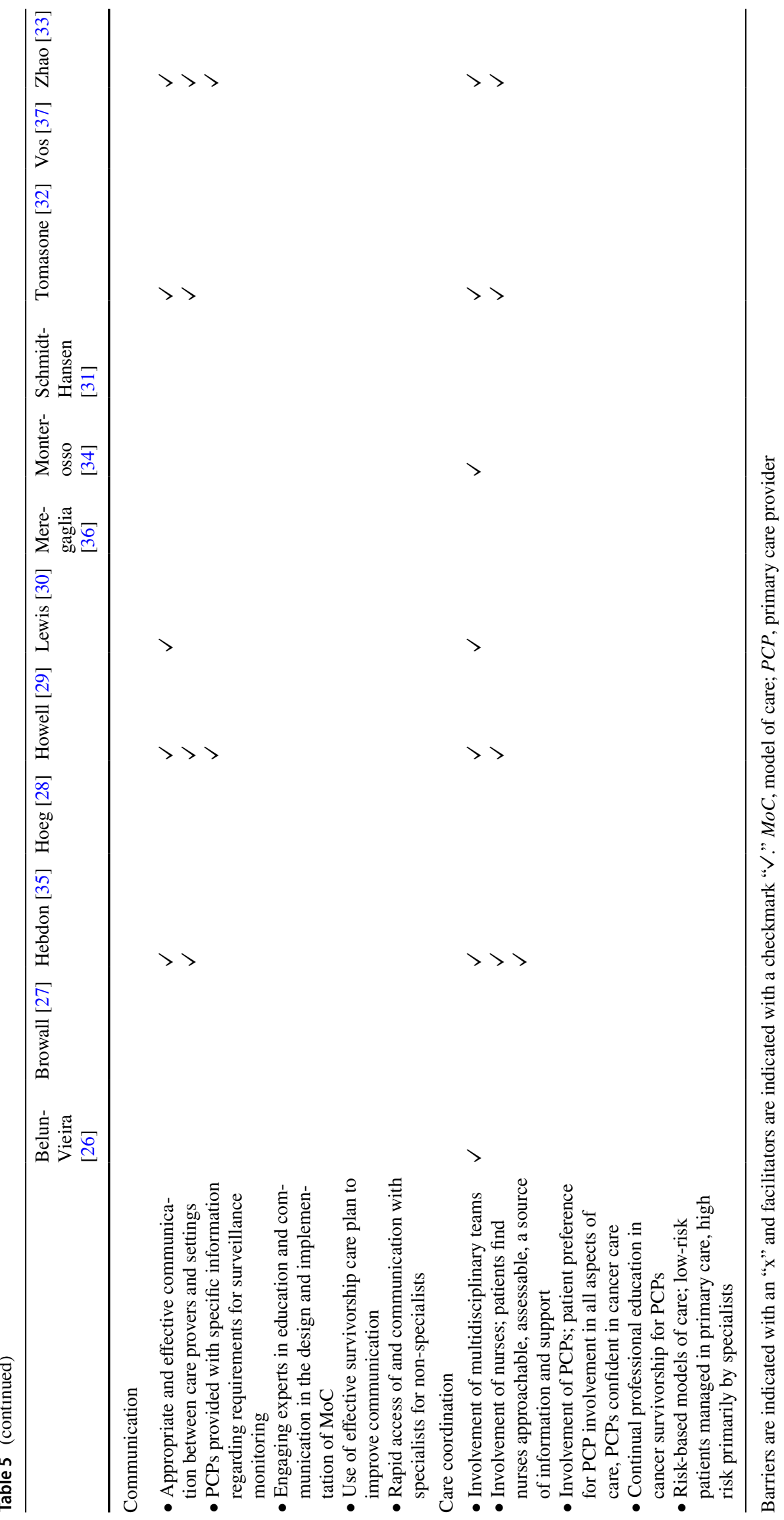




\section{Discussion}

This overview of systematic reviews is the first to synthesize and evaluate the highest quality (i.e., level I) evidence regarding the effectiveness of models of cancer care follow-up as well as implications for implementation. Overall, findings suggest that no existing data shows any differences between models of care for a range of healthcare outcomes and domains of quality cancer survivorship care. However, nurse-led and shared care models may have more economic benefits and utilize fewer healthcare resources than specialist-led care. Additionally, these models of care have been shown to increase patient acceptability and satisfaction due to improvements in convenience, shorter waiting, and travel times with their GP, improving quality of life as well as promoting continuity of care post-treatment $[34,40]$. As there is minimal current practical guidance regarding the implementation of models of care other than specialist-led models, there is significant scope for future research. The COVID-19 pandemic has shed new light on how cancer care is delivered, and it is possible that some learning from this will be relevant to the delivery of cancer survivors' care alternatives outside of specialist-led models [41]. Since COVID-19, models of cancer care have been adapted or implemented including the amplified use of telehealth models and other remote patient monitoring technologies that incorporate community resources, primary care and allied health disciplines, as well as clinics to keep cancer survivors away from acute care hospitals as much as possible [42, 43]. Many of these changes have been adopted successfully to deliver healthcare services globally and could be integrated within routine cancer care beyond the duration of the pandemic.

Importantly, existing evidence suggests that no other models of follow-up cancer care have a confirmed negative impact on cancer survivor outcomes when compared to specialist-led care, and thus, if these options are more sustainable and cost-effective, they may be an appropriate method of delivering follow-up care. Transitioning away from specialist-led models of care and diverting follow-up care to other health professionals is envisaged to lead to increased capacity for oncology and hematology specialists to see more newly diagnosed patients sooner. This is key to ensuring the sustainability of the acute cancer care system as cancer incidences continue to rise. Accordingly, cancer survivors with a prior diagnosis of breast, colorectal, or prostate cancer or melanoma may be advised to access GPled, nurse-led, or shared care rather than specialist-led care where possible $[44,45]$. Although the current evidence suggests similar outcomes between different models of care in comparison with specialist-led care, the evidence does not contain all relevant outcomes. For instance, several important outcomes have not been measured or reported, including those related to the management of comorbid conditions, relationship difficulties, employment challenges, financial toxicity, and transitioning back to daily life. Additionally, the impact of different models of follow-up care on fear of cancer recurrence, which is one of the most common and debilitating concerns among cancer survivors post-treatment [46], was only reported in one review [28].

Another key gap in the literature is the clinical effectiveness of different models of care across diverse population groups. Details on race or ethnicity, education level, income, or geographic locations were not reported in any included review, and details regarding age were poorly reported. Furthermore, studies in this overview were conducted predominantly in females with breast and gynecological cancers. With the growing number of patients living with advanced or metastatic cancer who continue to be followed in oncology settings, examining outcomes of survivorship care is also needed. Therefore, future research should explore how social determinants of health and cancer characteristics may impact the effectiveness and suitability of various models of survivorship care. Additionally, the current evidence was limited mainly to developed countries with universal healthcare coverage and strong primary care systems; thus, models of care cannot be generalized to developing countries and other market-based healthcare systems. Thus, the effectiveness and contextual appropriateness of models of care other than specialist-led models in diverse population groups, age cohorts, healthcare settings, and geographic regions require further investigation. Health provider skills and confidence are keys to the transition away from specialist-led models of care for most cancer cohorts [47, 48]; however, a practitioner's confidence in managing breast cancer survivorship issues may be very different from confidence levels in providing quality survivorship care to people with rare cancer types [49]. Thus, research in diverse clinical settings allowing for distinct levels of provider expertise is required [7]. Such evidence on comparative effectiveness should also include implementation issues (e.g., availability of clinical tools and evidence-based point of care guidelines) and may eventually facilitate stakeholder buy-in and support uptake of alternate models of care.

Primary studies of the included reviews were conducted in a variety of countries with different healthcare systems ( $85 \%$ of primary studies outside of the USA), meaning there is significant variation in insurance coverage, available publicly funded services, and allocation of resources to determine an appropriate model of care. As a result, economic evaluations of models of care need to carefully consider the local context and financial structures across different countries. In order to consider which models of survivorship care may be appropriate in particular contexts, the characteristics, advantages, and disadvantages of different models have 
been described by the American Society of Clinical Oncology (ASCO) [50]; however, this is very US focused and includes providers and services that do not exist in other settings. A more risk-stratified, personalized model which can be adapted to a given setting may also provide a solution, where oncology and supportive care professionals need to identify not only the individual needs of each patient but also the services provided by their local communities [7, 51]. For example, the United Kingdom National Health Service offers different pathways of cancer follow-up care with most breast, prostate, and colorectal cancer survivors going through a supported self-management pathway, the remainder accessing varying levels of shared or hospital-based care depending on risk of recurrence and long-term problems, and the presence and severity of physical and psychosocial issues [52].

This overview of systematic reviews has also broadly grouped the different models of care and it must be acknowledged that specific interventions or components of care within these groups may be heterogenous, restricting the ability to comment on specific elements of a follow-up care model which are most effective. Due to poor reporting of intervention characteristics, models of care elements that are likely to influence the effectiveness or implementation outcomes were unable to be explored further, such as the use of telemedicine, frequency of follow-up, content of followup care, or additional supports provided. Significant research has investigated the effect of survivorship care plans in cancer care [53]; however, it is important to acknowledge that survivorship care plans can enable documentation and communication within a wider model of care. These care plans should not be regarded as a single distinct intervention, but an essential communication tool within a model of care as a larger complex intervention. Different models of care and specific elements of care may be effective in different settings depending on individual needs, healthcare resources, and cancer survivors' clinical characteristics [18, 51]. Thus, it is imperative for health services to understand how specific follow-up care models and their characteristics may suit local resource and cultural contexts.

This overview also highlighted barriers to widespread implementation of different models of care which included health provider education and communication. The perceived need for subsequent cancer care training for primary care providers is one of the commonly cited barriers to shared care and primary care provider-led models [47]. Continual professional education may improve provider confidence and competence in cancer survivorship issues to improve the quality of care provision and uptake of shared care models [54]. Additionally, health provider education can assist to enhance the confidence of cancer survivors in their healthcare providers' cancer care competencies, which was also found to promote the acceptance and accessibility of other models of care beyond specialist-led care. Clear communication and patient-specific guidance could be an alternative approach to upskilling primary care providers and improving confidence [40]. However, it is important to consider that many trials did not include a training component as part of the intervention but still demonstrated similar outcomes. Furthermore, the perceived time constraints associated with enhanced communication practices and subsequent training is a major barrier to transitioning away from specialist-led care, but could be minimized with effective protocols, processes, and guidelines [32, 47].

\section{Limitations}

Care needs to be taken in the interpretation of findings, as significant results were often based on small numbers of primary studies. Additionally, a larger number of reviews examined nurse-led care, all addressed adult cancer survivors, and most primary studies focused on breast cancer, making it difficult to understand the efficacy of various models of care and diverse cancer populations. The inclusion criteria focusing on models of care for those who have completed primary cancer treatment may have excluded models of follow-up care for those living with advanced or metastatic cancers and/or others who are not being treated with curative intent. For these populations of patients, different models of care should be considered and evaluated. Another limitation is that included reviews also did not evaluate multidisciplinary cancer survivorship clinics as a model of care. Finally, the quality of two included SRs was considered critically poor for several reasons including a lack of study selection and data extraction in duplicate and not adequately assessing and accounting for the risk of bias. However, these reviews still provide valuable input on models of care with the quality rating not affecting the reported findings.

\section{Implications for future research}

There is currently a lack of evidence-based research to suggest which model of care is most effective, particularly in different cancer populations, and there are barriers to the generalizability of models of care from developed countries and cities to developing countries and rural settings. Future research is needed to expand the understanding of effective models of care in diverse cancer survivor populations including pediatric cancer survivors, adolescent and young adult (AYA) survivor groups [55], older adults [56], and a broader range of cancer types as well as advanced stages of the disease. Future studies should also prioritize robust primary studies to address gaps in the literature for outcomes in the domains of health promotion, chronic conditions, clinical structure, and decision-making. Addressing these gaps will help determine effective models of care using accurate 
measures [57]. Furthermore, future research must expand healthcare outcomes beyond the quality of life and execute additional robust economic evaluations for a wider range of models of care to provide evidence for health systems to fund and promote the transition to alternative models. Additionally, interventions tested in trials should be further tested in real-world settings, especially at the population level. Principles of implementation science can also be used to move from efficacy to effectiveness to cost-effectiveness and finally dissemination and implementation.

This overview has warranted a need for future studies that focus on implementation science and how access and uptake of models of care can be facilitated. In addition, the development of best practice guidelines including decision trees for selecting the most appropriate model of care for the local setting and individual cancer survivor, implementation guides, and standardized outcomes for the evaluation would be helpful to advance this field of science and practice. Future research evaluating models of care should be conducted with clear descriptions of the model of care elements and characteristics using validated tools to assess outcomes and to identify sustainable and viable alternatives to specialist-led care.

\section{Conclusion}

Since the IOM report [4], progress has been made in terms of developing several models of follow-up post-treatment care for cancer survivors [58, 59]; however, many efforts are still required to optimize the health and well-being of cancer survivors. This overview of systematic reviews suggests models of post-treatment follow-up cancer care delivered by nurses, primary care providers, or shared care models are acceptable and may have economic benefits over specialist-led care and that no models of follow-up cancer care have a negative impact on cancer survivor outcomes. Despite a high number of reviews regarding the effectiveness of models of care, significant gaps in important cancer survivor outcomes and different population groups remain. Additionally, as these models are not widely adopted, further economic evaluations and evidence-based recommendations to guide implementation are required. Cancer survivors are heterogeneous populations, thus providing appropriate post-treatment survivorship care is a complex process which requires a personalized, risk-based approach, and increased understanding of the required elements of effective models. Rather than aiming for an optimal "one-size fits all" model of survivorship care, care needs to be tailored to the individual, with appropriate models resulting in improved outcomes and healthcare efficiency.

Supplementary Information The online version contains supplementary material available at https://doi.org/10.1007/s11764-021-01128-1.
Author contribution All authors contributed to the study conception and design. Literature searches, data extraction, and reporting were performed by M.C., F.C.W., and R.J. The first draft of the manuscript was written by F.C.W. and all authors commented on previous versions of the manuscript. All authors read and approved the final manuscript.

Funding R.J.C. receives salary support from the National Health and Medical Research Council Investigator Grant (APP1194051). J.E. is supported by an NHMRC Investigator grant (APP1195302). This study was supported by the Primary Care Collaborative Cancer Clinical Trials Group (PC4).

Data availability All data generated or analyzed during this study are included in this published article and its supplementary files.

\section{Declarations}

Ethics approval Not applicable.

Consent to participate Not applicable.

Consent for publication Not applicable.

Conflict of interest The authors declare no competing interests.

\section{References}

1. Lisy K, et al. How do we define and measure optimal care for cancer survivors? An Online Modified Reactive Delphi Study. Cancers. 2021;13(10):2299. https://doi.org/10.3390/cancers131 02299.

2. Nekhlyudov, L., et al., Developing a quality of cancer survivorship care framework: implications for clinical care, research, and policy. JNCI: Journal of the National Cancer Institute. 2019; 111(11): 1120-1130. https://doi.org/10.1093/jnci/djz089

3. Mead $\mathrm{KH}$, et al. Identifying patients' priorities for quality survivorship: conceptualizing a patient-centered approach to survivorship care. J Cancer Surviv. 2020;14(6):939-58. https://doi.org/10. 1007/s11764-020-00905-8.

4. Hewitt M, Greenfield S, Stovall E. From cancer patient to cancer survivor: lost in transition. Washington, DC: National Academies Press; 2005.

5. Halpern MT, McCabe MS, Burg MA. The Cancer Survivorship Journey: Models of care, disparities, barriers, and future directions. Am Soc Clin Oncol Educ Book. 2016;35:231-9. https:// doi.org/10.1200/edbk_156039.

6. McCabe MS, et al. American Society of Clinical Oncology statement: achieving high-quality cancer survivorship care. J Clin Oncol. 2013;31(5):631. https://doi.org/10.1200/JCO.2012.46. 6854.

7. Nekhlyudov, L., M. O’Malley D, and S.V. Hudson, Integrating primary care providers in the care of cancer survivors: gaps in evidence and future opportunities. Lancet Oncol. 2017; 18(1): e30-e38. https://doi.org/10.1016/s1470-2045(16)30570-8

8. Alfano, C.M., et al., Implementing personalized pathways for cancer follow-up care in the United States: Proceedings from an American Cancer Society-American Society of Clinical Oncology summit. CA: A Cancer Journal for Clinicians. 2019; 69(3): 234-247. https://doi.org/10.3322/caac. 21558

9. Taylor K, Chan RJ, Monterosso L. Models of survivorship care provision in adult patients with haematological 
cancer: an integrative literature review. Support Care Cancer. 2015;23(5):1447-58. https://doi.org/10.1007/s00520-015-2652-6.

10. Mollica MA, et al. Follow-up care for breast and colorectal cancer across the globe: survey findings from 27 countries. JCO Global Oncology. 2020;6:1394-411. https://doi.org/10.1200/go.20. 00180.

11. Jefford M, et al. Implementing improved post-treatment care for cancer survivors in England, with reflections from Australia, Canada and the USA. Br J Cancer. 2013;108(1):14-20. https:// doi.org/10.1038/bjc.2012.554.

12. Rubin $\mathrm{G}$, et al. The expanding role of primary care in cancer control. Lancet Oncol. 2015;16(12):1231-72. https://doi.org/10. 1016/S1470-2045(15)00205-3.

13. Lewis, R., et al., Nurse-led vs. conventional physician-led follow-up for patients with cancer: systematic review. J Adv Nurs. 2009; 65(4): 706-723. https://doi.org/10.1111/j.1365-2648.2008. 04927.x

14. Chan RJ, et al. Breast cancer nursing interventions and clinical effectiveness: a systematic review. BMJ Support Palliat Care. 2020;10(3):276-86. https://doi.org/10.1136/bmjsp care-2019-002120.

15. Howell, D., et al., Management of cancer and health after the clinic visit: a call to action for self-management in cancer care. JNCI: Journal of the National Cancer Institute. 2020; 113(5): 523-531. https://doi.org/10.1093/jnci/djaa083

16. Ramsey I, et al. A core set of patient-reported outcomes for population-based cancer survivorship research: a consensus study. J Cancer Surviv. 2021;15(2):201-12. https://doi.org/10.1007/ s11764-020-00924-5.

17. Shapiro CL, et al. Quality care in survivorship: lessons learned from the ASCO Quality Oncology Practice Initiative. JCO Oncol Pract. 2021;17(8):e1170-80. https://doi.org/10.1200/op.21.00290.

18. Alfano CM, et al. Building personalized cancer follow-up care pathways in the United States: lessons learned from implementation in England, Northern Ireland, and Australia. Am Soc Clin Oncol Educ Book. 2019;39:625-39. https://doi.org/10.1200/ EDBK_238267.

19. Lisy K, et al. Identifying the most prevalent unmet needs of cancer survivors in Australia: a systematic review. Asia Pac J Clin Oncol. 2019;15(5):e68-78. https://doi.org/10.1111/ajco.13176.

20. Molassiotis A, et al. Mapping unmet supportive care needs, quality-of-life perceptions and current symptoms in cancer survivors across the Asia-Pacific region: results from the International STEP Study. Ann Oncol. 2017;28(10):2552-8. https://doi.org/10.1093/ annonc/mdx350.

21. Pollock, M., et al., Chapter V: Overviews of reviews, in J.T. Higgins, J, Editor. Cochrane handbook for systematic reviews of interventions: Cochrane; 2019.

22. Page MJ, et al. The PRISMA 2020 statement: an updated guideline for reporting systematic reviews. BMJ. 2021;372: n71. https:// doi.org/10.1136/bmj.n71.

23. Moher D, et al. Preferred reporting items for systematic review and meta-analysis protocols (PRISMA-P) 2015 statement. Syst Rev. 2015;4(1):1. https://doi.org/10.1186/2046-4053-4-1.

24. Shea BJ, et al. AMSTAR 2: a critical appraisal tool for systematic reviews that include randomised or non-randomised studies of healthcare interventions, or both. BMJ. 2017;358: j4008. https:// doi.org/10.1136/bmj.j4008.

25. Pieper D, et al. Systematic review finds overlapping reviews were not mentioned in every other overview. J Clin Epidemiol. 2014;67(4):368-75. https://doi.org/10.1016/j.jclinepi.2013.11. 007.

26. Belun-Vieira I, McInness D, Sheriff MK. In the case of prostate cancer patients, are there advantages in cancer nurse-led followup? Int J Urol Nurs. 2016;10(3):154-66. https://doi.org/10.1111/ ijun.12112.
27. Browall M, Forsberg C, Wengstrom Y. Assessing patient outcomes and cost-effectiveness of nurse-led follow-up for women with breast cancer - have relevant and sensitive evaluation measures been used? J Clin Nurs. 2017;26(13-14):1770-86. https:// doi.org/10.1111/jocn.13496.

28. Høeg, B.L., et al., Follow-up strategies following completion of primary cancer treatment in adult cancer survivors. Cochrane Database of Systematic Reviews. 2019; (11). https://doi.org/10. 1002/14651858.CD012425.pub2

29. Howell D, et al. Models of care for post-treatment follow-up of adult cancer survivors: a systematic review and quality appraisal of the evidence. J Cancer Surviv. 2012;6(4):359-71. https://doi. org/10.1007/s11764-012-0232-z.

30. Lewis RA, et al. Follow-up of cancer in primary care versus secondary care: systematic review. $\mathrm{Br} \mathrm{J}$ Gen Pract. 2009;59(564):e234-247. https://doi.org/10.3399/bjgp09X453567.

31. Schmidt-Hansen M, Baldwin DR, Hasler E. What is the most effective follow-up model for lung cancer patients? A systematic review. Journal of Thoracic Oncology: Official Publication of the International Association for the Study of Lung Cancer. 2012;7(5):821-4. https://doi.org/10.1097/JTO.0b013e31824afc55.

32. Tomasone JR, et al. Interventions to improve care coordination between primary healthcare and oncology care providers: a systematic review. Esmo Open. 2016;1(5): e000077. https://doi.org/ 10.1136/esmoopen-2016-000077.

33. Zhao Y, Brettle A, Ling Q. The effectiveness of shared care in cancer survivors-a systematic review. International Journal of Integrated Care (IJIC). 2018;18(4):1-17. https://doi.org/10.5334/ ijic.3954.

34. Monterosso L, et al. Systematic review and meta-analysis of patient reported outcomes for nurse-led models of survivorship care for adult cancer patients. Cancer Treat Rev. 2019;73:62-72. https://doi.org/10.1016/j.ctrv.2018.12.007.

35. Hebdon M, et al. Transitioning patients to survivorship care: a systematic review. Oncol Nurs Forum. 2014;41(6):615-25. https:// doi.org/10.1188/14.ONF.615-625.

36. Meregaglia M, Cairns J. Economic evaluations of follow-up strategies for cancer survivors: a systematic review and quality appraisal of the literature. Expert Rev Pharmacoecon Outcomes Res. 2015;15(6):913-29. https://doi.org/10.1586/14737167.2015. 1087316.

37. Vos JAM, et al. Survivorship care for cancer patients in primary versus secondary care: a systematic review. J Cancer Surviv. 2020;19:19. https://doi.org/10.1007/s11764-020-00911-w.

38. Haggstrom DA, et al. Follow-up care delivery among colorectal cancer survivors most often seen by primary and subspecialty care physicians. J Gen Intern Med. 2009;24(2):472. https://doi.org/10. 1007/s11606-009-1017-6.

39. Wattchow DA, et al. General practice vs surgical-based follow-up for patients with colon cancer: randomised controlled trial. $\mathrm{Br} \mathbf{J}$ Cancer. 2006;94(8):1116-21. https://doi.org/10.1038/sj.bjc.66030 52.

40. Lisy, K., et al., Sharing cancer survivorship care between oncology and primary care providers: a qualitative study of health care professionals' experiences. J Clin Med. 2020; 9(9). https://doi.org/ $10.3390 / \mathrm{jcm} 9092991$

41. Thomson CA, et al. Addressing cancer survivorship care under COVID-19: perspectives from the cancer prevention and control research network. Am J Prev Med. 2021;60(5):732-6. https://doi. org/10.1016/j.amepre.2020.12.007.

42. Koczwara B. Cancer survivorship care at the time of the COVID19 pandemic. Med J Aust. 2020;213(3):107-108.e101. https://doi. org/10.5694/mja2.50684.

43. Underhill C, et al. Towards new models of cancer care in Australia: lessons from Victoria's response to the COVID-19 
pandemic. Intern Med J. 2020;50(10):1282-5. https://doi.org/10. 1111/imj.15012.

44. Jefford, M., et al., The important role of general practice in the care of cancer survivors. Aust J Gen Pract. 2020; 49(5): 288-292. https://doi.org/10.31128/ajgp-10-19-5133

45. Bessen T, et al. What sort of follow-up services would Australian breast cancer survivors prefer if we could no longer offer longterm specialist-based care? A discrete choice experiment. Br J Cancer. 2014;110(4):859-67. https://doi.org/10.1038/bjc.2013. 800.

46. Smith AB, et al. Spotlight on the fear of cancer recurrence inventory (FCRI). Psychol Res Behav Manag. 2020;13:1257-68. https://doi.org/10.2147/prbm.S231577.

47. Lisy K, et al. Facilitators and barriers to shared primary and specialist cancer care: a systematic review. Support Care Cancer. 2021;29(1):85-96. https://doi.org/10.1007/s00520-020-05624-5.

48. Chan RJ, et al. Oncology practitioners' perspectives and practice patterns of post-treatment cancer survivorship care in the Asia-Pacific region: results from the STEP study. BMC Cancer. 2017;17(1):715. https://doi.org/10.1186/s12885-017-3733-3.

49. Del Giudice ME, et al. Primary care physicians' views of routine follow-up care of cancer survivors. J Clin Oncol. 2009;27(20):3338-45. https://doi.org/10.1200/jco.2008.20.4883.

50. American Society of Clinical Oncology (ASCO). Models of longterm follow-up care. Survivorship Compendium https://www. asco.org/news-initiatives/current-initiatives/prevention-survivorsh ip/survivorship-compendium/models-long. Accessed 04 Jun 2021.

51. Mayer, D.K. and C.M. Alfano, Personalized risk-stratified cancer follow-up care: its potential for healthier survivors, happier clinicians, and lower costs. JNCI: Journal of the National Cancer Institute. 2019; 111(5): 442-448. https://doi.org/10.1093/jnci/djy232

52. NHS Improvement. Stratified pathways of care....From conception to innovation. $2011 \mathrm{https} / / / \mathrm{www}$.england.nhs.uk/impro
vement-hub/wp-content/uploads/sites/44/2017/11/StratifiedPathways-of-Care.pdf Accessed 04 Jun 2021.

53. Hill RE, et al. Survivorship care plans in cancer: a meta-analysis and systematic review of care plan outcomes. Oncologist. 2020;25(2):e351-72. https://doi.org/10.1634/theoncologist. 2019-0184.

54. Chan RJ, et al. Outcomes of cancer survivorship education and training for primary care providers: a systematic review. J Cancer Surviv. 2021. https://doi.org/10.1007/s11764-021-01018-6.

55. Tonorezos ES, et al. Models of care for survivors of childhood cancer from across the globe: advancing survivorship care in the next decade. J Clin Oncol. 2018;36(21):2223-30. https://doi.org/ 10.1200/jco.2017.76.5180.

56. Mohile SG, et al. Improving the quality of survivorship for older adults with cancer. Cancer. 2016;122(16):2459-568. https://doi. org/10.1002/cncr.30053.

57. Halpern MT, Argenbright KE. Evaluation of effectiveness of survivorship programmes: how to measure success? Lancet Oncol. 2017;18(1):e51-9. https://doi.org/10.1016/s1470-2045(16) 30563-0.

58. Nekhlyudov L, et al. Going beyond being lost in transition: a decade of progress in cancer survivorship. J Clin Oncol. 2017;35(18):1978-81. https://doi.org/10.1200/jco.2016.72.1373.

59. Kline, R.M., et al., Long-term survivorship care after cancer treatment - summary of a 2017 National Cancer Policy Forum Workshop. JNCI: Journal of the National Cancer Institute. 2018; 110(12): 1300-1310. https://doi.org/10.1093/jnci/djy176.

Publisher's note Springer Nature remains neutral with regard to jurisdictional claims in published maps and institutional affiliations.

\section{Authors and Affiliations}

\section{Raymond J. Chan ${ }^{1,2,3}$ (D) . Fiona Crawford-Williams ${ }^{1,3} \cdot$ Megan Crichton $^{3,4} \cdot$ Ria Joseph $^{3} \cdot$ Nicolas H. Hart $^{1,3,5,6}$. Kristi Milley ${ }^{7,8} \cdot$ Paige Druce $^{7,8} \cdot$ Jianrong Zhang ${ }^{8} \cdot$ Michael Jefford $^{9,10} \cdot$ Karolina Lisy $^{9,10} \cdot$ Jon Emery $^{7,8}$. Larissa Nekhlyudov ${ }^{11,12}$}

1 Caring Futures Institute, College of Nursing and Health Sciences, Flinders University, Adelaide, South, Australia

2 Division of Cancer Services, Princess Alexandra Hospital, Brisbane, QLD, Australia

3 Cancer and Palliative Care Outcomes Centre, School of Nursing, Queensland University of Technology, Brisbane, QLD, Australia

4 Bond University Nutrition and Dietetics Research Group, Faculty of Health Science \& Medicine, Bond University, Robina, QLD, Australia

5 School of Medical and Health Sciences, Edith Cowan University, Perth, WA, Australia

6 Institute for Health Research, University of Notre Dame Australia, Perth, WA, Australia
7 Primary Care Collaborative Cancer Clinical Trials Group (PC4), Carlton, VIC, Australia

8 Centre for Cancer Research and Department of General Practice, University of Melbourne, Melbourne, VIC, Australia

9 Department of Oncology, Sir Peter MacCallum, University of Melbourne, Parkville, VIC, Australia

10 Australian Cancer Survivorship Centre, Peter MacCallum Cancer Centre, Melbourne, VIC, Australia

11 Department of Medicine, Harvard Medical School, Boston, MA, USA

12 Department of Medicine, Brigham and Women's Hospital, Harvard Medical School, Boston, MA, USA 